\title{
Assisted hatching in assisted reproduction: a state of the art
}

\author{
Mohamad Eid Hammadeh • \\ Constanze Fischer-Hammadeh $\cdot$ Khaled Refaat Ali
}

Received: 16 August 2010 / Accepted: 11 October 2010 /Published online: 2 November 2010

(C) Springer Science+Business Media, LLC 2010

\begin{abstract}
The World Health Organization estimates that one in six couples experience some delay in conception and an increasing number require treatment by the assisted conception (AC) procedures of in vitro fertilization (IVF) or intracytoplasmic sperm injection (ICSI).The implantation rate of embryos resulting from in vitro fertilization cycles is generally less than $20 \%$. The exposure of oocytes and embryos to the artificial conditions of in vitro culture may have negative effects on the embryo's ability to undergo normal hatching, resulting in low rates of implantation following IVF and embryo transfer. Human embryos resulting from superovulation develop more slowly in vitro compared to embryos in vivo, manifest a relatively high degree of cytogenetic abnormalities and undergo cellular fragmentation. Artificially disrupting the zona pellucida is known as assisted hatching ( $\mathrm{AH})$ and there is some evidence that embryos that have undergone zona manipulation for assisted hatching tend to implant one day earlier than unhatched embryos. A variety of techniques have since been employed to assist embryo hatching, including partial mechanical zona dissection, zona drilling and zona thinning, making use of acid tyrodes, proteinases, piezon
\end{abstract}

Capsule This review will consider the impact of IVF conditions on zona pellucida physiology, zona hardening, different techniques of assisted hatching, who may benefit from assisted hatching and potential hazards

M. E. Hammadeh $(\bowtie) \cdot$ C. Fischer-Hammadeh

Obstetrics and Gynaecology Department, Assisted Reproduction

Technology Unit, University of Saarland,

Homburg/Saar, Germany

e-mail: mehammadeh@yahoo.de

K. R. Ali

Obstetrics and Gynaecology Department, Al-Azhar University, Assuit, Egypt vibrator manipulators and lasers. This review will consider the impact of IVF conditions on zona pellucida physiology, zona hardening, different techniques of assisted hatching, who may benefit from assisted hatching and potential hazards.

Keywords Assisted hatching - Zona pellucida .

Implantation · LASER · Zona drilling · Tyrode's solution

\section{Introduction}

Despite numerous developments in IVF and ICSI, the implantation rate of the replaced embryos remains low, and it has been estimated that up to $85 \%$ of replaced embryos do not implant [1]. It has been reported that implantation rate per embryo transfer in IVF/ICSI programs is $10 \%$ to $15 \%$ for day 2 or day 3 transfers and $23 \%$ to $25 \%$ for blastocyst transfers. The ability of an embryo to develop and implant primarily relates to the quality of originating gametes and intrinsic characteristics of the embryo, such as its chromosomal constitution and the quality of its cytoplasm [2]. However, some proportions of euploid embryos with full developmental potential fail to implant because of hatching difficulties [2]. Numerous approaches to improve the implantation rate have been proposed and practiced. These include [1] improving the technique of embryo transfer, [3] improving endometrial receptivity, and [4] improving the capacity of the embryo to implant. Assisted hatching (AH) has been proposed as a method for improving the capacity of the embryos to implant. Assisted hatching involves artificial disruption of the zona pellucida, and a variety of $\mathrm{AH}$ techniques have been employed including zona thinning, zona drilling (breaching by forming a hole) and complete removal of the zona, use of 
chemicals, other mechanical techniques, and the use of lasers [5]. In the past decade, AH has been offered to older patients as well as to those with frozen-thawed embryos or who have suffered recurrent IVF-ET failure. The effect of $\mathrm{AH}$, however, is still debatable [6-9]. Assisted hatching was first described by Cohen et al. who reported the first pregnancy after assisted hatching in 1988 [10]. This pioneering work was followed by numerous publications. Some of these publications reported a significant increase in the pregnancy and/or implantation rate in all patients [11]; other publications reported significant improvement in patients with a poor prognosis, namely women over 38 years of age, patients with thick zonae, and patients with repeated implantation failures [12-14]; while a third group of publications reported a non significant improvement in the pregnancy rate in patients with poor prognosis $[11,15-18]$. On the contrary, some publications reported no improvement in pregnancy or implantation [19-21].

\section{Zona pellucida structure and function}

The human oocyte and early embryo is surrounded by $13-$ $15 \mu \mathrm{m}$ thick acellular matrix, the zona pellucida [22], which is composed of glycoproteins, carbohydrates and zona pellucida-specific proteins. The zona pellucida is bilayered, the outer is thick, whereas the inner is thin but resilient [3]. The zona pellucida is of structural and functional importance during fertilisation and preimplantation development. It is involved in sperm binding, induction of the acrosome reaction and it promotes egg fusion [23]. Following fertilisation, the zona pellucida blocks polyspermy, prevents blastomeres dispersal and helps in oviductal transport.It also avoids contact with other cells (epithelial lining of the reproductive tract, leucocytes, spermatozoa and other cells of the embryo) [24].It is essential for maintaining the integrity of the precompacted embryo. Compaction is the formation of the structural junctions between the blastomeres. Once compaction occurs, the zona pellucida is no longer essential [25].

To ensure the above function, zona hardening naturally occurs, and is evidenced by an increased resistance to dissolution by different chemical agents [22].

\section{Hatching process}

Upon reaching the blastocyst stage, a combination of lysins 'proteases' produced by the cleaved embryo (trophoectoderm) and/or the uterus, all assist in zona dissolution [26-28].

Moreover, the physical expansion of the embryonic mass reduces the zona thickness in preparation for hatching [29], so that the trophoectoderm cells interact with endometrial cells and implantation occurs. Hatching of the embryo is a process, whereby the expanded blastocyst undergoes cycles of contractions and expansions until it becomes almost invisible [30]. Elasticity and thinning of the zona pellucida are fundamental for successful hatching, which is a prerequisite for implantation [29].

There are two observational studies demonstrating that a natural hatching site usually develops in close proximity to the inner cell mass (ICM) of blastocysts in humans, whereas that of the mouse is at the side opposite to the ICM [31, 32]. So Hirotoshi et al., hypothesized that the choice of $\mathrm{AH}$ site could be important to the development of hatching [33].

The rationale for assisted hatching

An important observation leading to the clinical introduction of assisted hatching was the finding that microsurgically fertilised embryos with artificial gaps in their zona appear to have high rates of implantation [34], and that cleaved embryos with a good prognosis for implantation have reduced zona thickness [35].

Further, Wright et al. [36] reported that some embryos displayed variation in zona thickness at their early stage of development and that zona thinning is an active process. In contrast, zygotes that did not cleave did not show any change in zona pellucida thickness in culture [37]. The zona pellucida thickness has been shown to be correlated with the age of the patient by some [38] but not others [39]. Failure of zona rupture and subsequent impaired hatching have thus been suggested to account at least in part for the relatively low implantation rates following assisted reproductive techniques [40].

Embryos may intrinsically have a thick zona pellucida ( $>15 \mathrm{Am}$ ), or a secondary hardening of the zona pellucida may be suspected to occur during in vitro culture and after cryopreservation [39].

The relationship between zona thickness and implantation has been queried, suggesting that the limiting factor for successful hatching is not the overall thickness but the resilience of the thin inner layer [3]. Moreover, no correlation exists between zona pellucida thickness and zona pellucida hardness [39]. As a result, chemical removal of the outside of the zona pellucida of the Day 3 embryo has no impact on implantation rate, whereas the creation of a hole in the zona pellucida may improve embryo implantation [15]. As opposed to zona thickness and zona hardness, the rationale for performing assisted hatching procedure may be to overcome a deficiency in the production of an intrinsic embryonic lysin that promotes hatching following blastocyst expansion [39]. Schiewe et al. reported that suboptimal culture conditions may therefore account for a qualitative impairment of zona lysin production. Thus, optimising culture conditions might overcome such in vitro impairment [39]. They also showed 
that some embryos may experience a reduction or even a complete inability to secrete the 'hatching factor' (a quantitative impairment), independent of culture conditions or possible uterine lysin, which may inhibit the normal hatching. However, the concept of a uterine factor remains elusive due to the lack of direct evidence. In this situation, artificial thinning, or opening the zona pellucida in an earlier developmental stage might increase the incidence of hatching.

\section{Mechanism of AH}

Although the mechanism by which assisted hatching promotes embryo implantation remains unclear, there are possible explanations. The implantation window is the critical period when the endometrium reaches its ideal receptive state for implantation. The majority of embryos hatch during this time, suggesting that a precise synchronization between the embryo and the endometrium is essential [41].

Early implantation has been associated with subsequent viable pregnancy, whereas delayed implantation has been associated with a high incidence of abortion [42]. Embryos with artificial gaps in their zonae initiate hatching earlier than zonae intact embryos [43]. Therefore, it can be postulated that assisted hatching facilitates implantation by allowing earlier embryo-endometrium contact. Further, although most molecules are able to cross the zona pellucida, the rate of transport may be related to zona thickness. A two-way transport of metabolites and growth factors across the zona pellucida may be altered due to the presence of the artificial gap [15, 34]. Such contact may permit earlier exposure of the embryos to vital growth factors. For the mechanism of $\mathrm{AH}$ enhancement, the experimental data in the mouse model has demonstrated increasing embryo blastocyst formation (on day 5) in a hatched group (on day 3) compared with control. Theoretically, AH opens important routes to convey nutrients from the incubating media. These nutrients enhance embryo development and blastocyst formation [44].

Techniques of assisted hatching

Since the first assisted hatching technique that used a mechanical method, several approaches have been proposed [45], including mechanical incision of the zona [43]; chemical zona drilling with acidic medium [46]; chemical zona thinning [47]; laser-assisted hatching [33] and, more recently, piezo technology [14]. The mechanical and the chemical methods require extensive technical skill to produce uniform, well controlled and standardised micro holes using micropipettes mounted on micromanipulators. It is important to minimise the time the embryo is out from the incubator and to optimise the methodologies to reduce
$\mathrm{pH}$ and temperature variations that can be detrimental to embryo development. Micromanipulation is done on Day 2 or later (after the adherence between the blastomeres has increased), on embryos that are chosen for replacement.

\section{Mechanical techniques}

The technique of assisted hatching using partial zona dissection (PZD) to create an artificial opening of the zona pellucida of early cleaved embryos was first described by Cohen et al. [46]. While the embryo is stabilised by a holding pipette, the zona pellucida is pierced with a microneedle that is pushed tangentially through the space between the zona pellucida and blastomeres until it pierces through the zona pellucida again. The embryo is released from the holding pipette. The small part of the zona trapped against the microneedle is then rubbed against the holding pipette, thus opening the area between the two sides pierced by the microneedle. The mechanism of PZD is quick to perform, but it produces holes of variable sizes that may not always be optimal [48]. A refinement of PZD has been described where a second cut is made in the zona pellucida under the first cut at a right angle, leaving a cross-shaped hole on the surface of the zona pellucida (three-dimensional PZD) [49]. This method allows the creation of larger openings while permitting the protection of the embryo by the zona pellucida flaps during embryo transfer. Nijs et al. [50] described a zona rubbing technique to assisted hatching, by reducing the thickness of the matrix through gentle rubbing with a microneedle. No gap is made in the zona pellucida, so the risk of blastomere loss and invasion of micro-organisms or immune cells is minimised. However, care should be taken when transferring double or triple embryos following this procedure for fear of increasing the incidence of multiple pregnancies [51].

\section{Mechanically expanding the zona pellucida}

This technique was inspired by the natural expanding effects of blastocysts on the ZP. This mechanical AH neither thins nor breaches the ZP; rather, it expands/ stretches the $\mathrm{ZP}$ via the injected hydrostatic pressure to assist with the embryo hatching process. A recent study, was done by applying this new method to frozen-thawed day-3 (D3) embryos to determine whether it could enhance their implantation ability. It was assumed that ZP hardening in frozen-thawed embryos is more pronounced than in fresh embryos. It stated that mechanically expanding the ZP of frozen-thawed D3 embryos with injected hydrostatic pressure after thawing increases the implantation rate compared with control embryos [52].

Cong Fang and colleagues from the First Affiliated Hospital of Sun Yat-Sen University, Guangdong, People's 
Republic of China, conducted the study in subjects who had undergone their first IVF/ICSI cycles (2006-2008) to assess whether a new $\mathrm{AH}$ technique improves the rates of implantation and clinical pregnancy. The patients underwent thawed embryo transfer and were randomly assigned into two groups. In the $\mathrm{AH}$ group: Embryos were transferred after injected hydrostatic pressure was used to expand the ZP of D3 cryopreserved and thawed embryos. In the group without $\mathrm{AH}$ (controls): ICSI needles were used to pierce the embryos, but not expand the ZP. The embryos were thus transferred without performing $\mathrm{AH}$.

The researchers, however, reported higher rates of implantation and clinical pregnancy in the AH group compared to the controls, despite the transfer of the same number of embryos. The study concluded that mechanical expansion of the ZP is a potentially safe and effective method to improve the outcome [52].

\section{Chemical techniques}

Assisted hatching using acid Tyrode's has been described in detail by Cohen et al. $[15,46]$. The embryo is secured on a holding pipette and a microneedle is applied to an area of the zona pellucida overlying either empty perivitelline space or extracellular fragments (i.e. a blastomere-free area). The microneedle is preloaded with Tyrode's acid before each micromanipulation using mouth-controlled suction. The acidic solution is expelled gently over a small area until the zona is breached. Suction is applied immediately after breaching the zona pellucida to prevent excess acid entering the perivitelline space. This technique requires very quick handling in order to avoid unnecessary exposure of the embryo to the acidic solution. The acid may be detrimental to the blastomeres adjacent to the drilled part of the zona pellucida. The zona dissolves on contact with the acid, hence, the embryo is removed immediately and rinsed several times to remove any trace of the acid.

Cruciate thinning of the zona pellucida with acid Tyrode's has been described in mice and humans [3]. It involves a biaxial application of the acid to make a cruciate area of thinning over about one-third of the circumference of the zona pellucida, without creating an opening. Embryos are washed free of the acid Tucker et al.[3], in their study, were unable to find this method effective, concluding that the bilayered human zona pellucida needs to be fully breached unlike that of the mouse.

\section{Piezo technology}

Recently, piezo technology has been introduced for zona pellucida drilling [14]. While a holding pipette holds the embryo, vibratory movements produced by a piezo-electric pulse regulated by a controller are used to carve a limited conical area in the zona pellucida. Five to eight applications in adjacent areas may be used to produce a large hole, which facilitates the complete hatching of blastocyst.

Laser-assisted hatching

Laser-assisted zona drilling was first reported in 1991 by two groups led by Tadir [53] and Palankar [54]. Laser presents an ideal tool for microsurgical procedures, as the energy is easily focussed on the targeted area producing a controlled and precise hole consistent between operators. The laser beam may either be directed using an optical lens tangential to the embryo through the zona pellucida in a non-contact mode or the laser may be guided through an optical fibre touching the embryo in a contact mode. Initially, non contact mode was adopted for gamete micromanipulation using various laser wavelengths. Subsequently, the contact approach was used with ultraviolet (UV) wavelength delivered by glass pipette, or infrared (IR) wavelength delivered with a quartz fibre [53]. However, the technical advantages of the non-contact mode and the potential of UV radiation to cause harmful mutagenic effects have led to the general preference for non-contact mode using the IR $1.48 \mu \mathrm{m}(1,480 \mathrm{~nm})$ diode laser [55].

Initially, the laser was used to create a single full thickness hole through the zona pellucida. More recently, Blake et al.[56] demonstrated a significant increase in hatching in vitro, using the laser to thin the zona pellucida without creating a hole. A further study found a higher clinical pregnancy rate when using the laser to thin an extended area of the zona pellucida compared with producing a single full thickness hole [57]. Both light electron microscopy and scanning electron microscopy revealed no ultrastructural degenerative alterations of the zona pellucida of oocytes and embryos following laser assisted zona drilling [58], and reports of the children delivered following this technique have been reassuring [59, 60]. Laser assisted microdissection of the ZP can be done with high precision and repeatability with no negative impact on in vitro embryo development. The technique is easy to perform and very effective with regard to the overall time requirement and can be performed in a sterile environment without any additional micromanipulations [61-65] by using the infrared $1.48-\mu \mathrm{m}$ diode laser it is feasible to open the zona even in largely expanded blastocysts without visible blastocyst damage. The safety of the $1.48-\mu \mathrm{m}$ diode laser beam has been evaluated in mouse and human oocytes and zygotes.

\section{Clinical application of $\mathrm{AH}$}

It is important to focus on who may benefit from this technique as several studies have shown no increase in 
pregnancy rates when assisted hatching is performed on unselected groups (Table 1).

However, there are factors have been suggested to identify groups of patients who may benefit from assisted hatching, including the number of previous IVF failures, the patient's age, replacement of cryopreserved embryos and the zona pellucida thickness. The variations in the techniques used, patients selected and study designs make it difficult to compare between studies and pool data

\section{The potential of AH in recurrent IVF failure}

Repeated unexplained IVF failure is associated with a reduced chance of pregnancy in subsequent treatment cycles. Pregnancy and implantation rates appear to be significantly increased by assisted hatching for women with unexplained recurrent implantation failure in at least two, and usually more, previous IVF cycles. However, it would of course be preferable to identify patients who would benefit from assisted hatching before they have unsuccessful IVF cycles [70] (Figs. 1, 2)

The potential of AH in Advanced female age

It is well established that IVF success rates decrease with advancing maternal age and many suggestions have been made to explain this reduction in fertility [71]. One such suggestion is that there may be spontaneous zona hardening due to age-related endocrine changes and/or the absence of lysins from surrounding tissues, which may act on embryos in vivo $[17,72]$.

Such environmental conditions either control the hatching important to recognize that there may be other factors reducing fertility in older women such as reduced quality and quantity of oocytes 'ovarian reserve' [71], an increase in the incidence of karyotypically abnormal embryos [15] may be spontaneous zona hardening due to age-related endocrine changes and/or the absence of lysins from surrounding tissues, which may act on embryos in vivo [17, 72]. Such environmental conditions either control the hatching Nevertheless, it is and to some extent, a decline in uterine receptivity $[16,73]$. Although assisted hatching may benefit older women, it is thus unlikely to restore their pregnancy rate to that seen in younger women (Table 2).

\section{Frozen-thawed embryos}

Excessive in vitro culture of cryopreserved embryos, and/or exacerbated by the freeze-thaw process, is thought to induce alteration in the glycoprotein matrix leading to zona hardening [77]. Check et al. [78] reported on improved implantation and pregnancy rates when assisted hatching was performed on six to eight cell stage embryos. Also, a successful outcome of assisted hatching when used on frozen-thawed two cell stage embryos was reported by Tao and Tamis [79].

Nijs et al. [51] observed significant increase of implantation rate after transfer of frozen-thawed embryos following application of zona rubbing. Further, a prospective randomised controlled study by Nagy et al. [80] presented a significant increase in implantation and pregnancy rates following laser-assisted hatching and removal of degenerated blastomeres, thawed two cell stage embryos was reported by Tao and Tamis [79].

Thick zona pellucida

A study of assisted hatching for patients with thick zona pellucida more than $15 \mu \mathrm{m}$ suggested an im-

Table 1 Studies of assisted hatching in unselected patients following IVF

\begin{tabular}{|c|c|c|c|c|c|c|c|c|c|}
\hline \multirow[t]{2}{*}{ Type of AH } & \multicolumn{2}{|c|}{ No. of patients } & \multicolumn{3}{|c|}{ Implantation rat (\%) } & \multicolumn{3}{|c|}{ Pregnancy rates (\%) } & \multirow[t]{2}{*}{ Ref. } \\
\hline & $\mathrm{AH}$ & Control & $\mathrm{AH}$ & Control & $P$ & $\mathrm{AH}$ & Control & $P$ & \\
\hline PZD & 15 & 15 & 25 & 6 & $<0.023$ & 47 & 13 & $<0.05$ & [47] \\
\hline Acid Tyrode's & 69 & 68 & 28 & 21 & NS & 54 & 47 & NS & [15] \\
\hline Acid Tyrode's & 110 & 108 & 18.9 & 21.9 & NS & 44.6 & 37 & NS & [3] \\
\hline PZD after ICSI & 60 & 60 & 17.9 & 17.1 & NS & 42.1 & 38.1 & NS & [66] \\
\hline Laser & 111 & 121 & 11.8 & 7.1 & $<0.05$ & 39.6 & 19 & $<0.05$ & [4] \\
\hline Acid Tyrode's & 13 & 7 & 9.6 & 10.7 & NS & 23 & 42 & NS & [67] \\
\hline Laser & 96 & 103 & - & - & - & 30.2 & 29.1 & NS & [68] \\
\hline Laser after ICSI & 51 & 52 & 17.3 & 20.8 & NS & 33.3 & 40.3 & NS & [69] \\
\hline Acid Tyrode's after ICSI & 27 & 25 & - & - & - & 44 & 40 & NS & [11] \\
\hline
\end{tabular}




\begin{tabular}{|c|c|c|c|c|c|}
\hline Study & $\begin{array}{l}\text { Assisted hatching } \\
n / \mathbb{N}\end{array}$ & $\begin{array}{l}\text { No hatching } \\
\mathbf{n} / \mathbf{N}\end{array}$ & $\begin{array}{c}\text { OR } \\
(95 \% \mathrm{Cl} \text { Fixed })\end{array}$ & $\begin{array}{l}\text { Weight } \\
\%\end{array}$ & $\begin{array}{l}\text { OR } \\
\text { (95\%CI Fixed) }\end{array}$ \\
\hline Antinori et al (12) & 32.172 & $19 / 98$ & - & 24.0 & $3.33[1.68,6.59]$ \\
\hline Chao et al (18) & $14 / 33$ & $5 / 31$ & & 8.0 & $3.83[1.18,12.47]$ \\
\hline Magli et al (13) & $45 / 135$ & $14 / 110$ & - & 27.6 & $3.43[1.76,6.67]$ \\
\hline Nakayama et al (14) & $17 / 126$ & $7 / 122$ & $\longrightarrow$ & 16.5 & $2.56[1.02,6.42]$ \\
\hline Stein et al (77) & $15 / 72$ & $12 / 82$ & $\longrightarrow$ & 23.9 & $1.54[0.67,3.54]$ \\
\hline Total(95\% $9 \mathrm{Cl})$ & $123 / 438$ & $57 / 443$ & - & 100.0 & $2.84[1.99,4.06]$ \\
\hline \multicolumn{6}{|c|}{ Test for heterogeneity chi-square $=2.89 d f=4 p=0.58$} \\
\hline
\end{tabular}

Fig. 1 Tree diagram for pregnancy rates in patients with $>2$ previous IVF failures undergoing assisted hatching versus those with no assisted hatching

proved implantation rate [15]. This has led some to introduce selective assisted hatching on poor prognosis embryos (thick zona pellucida, low developmental rate and excessive fragmentation), but further studies are required.

\section{Results of $\mathrm{AH}$}

The routine or universal performance of assisted hatching on all embryos in IVF/ICSI patients is neither scientific nor appropriate. Randomized trials of assisted hatching on all embryos without any selection, revealed that there is no difference in the implantation and pregnancy rates between the treatment and control groups [7, 66].

In a recently published meta-analysis of randomized controlled studies on assisted hatching for all patients treated with IVF/ICSI (13 studies out of 165 fitted the selection criteria); the results of the meta-analysis could not be accepted because of the heterogeneity of the studies which may be due to the different techniques used or due to different patient populations studied [70]. On the other hand, there is convincing evidence in the literature that assisted hatching may increase the implantation capability of some of the embryos. The proposed indications for assisted hatching are advanced maternal age ( $\geq 37$ years), elevated basal FSH of women and two or more previously failed IVF attempts, embryos with thick zona pellucida (>15 $\mu \mathrm{m})$, abnormal or poor embryo morphology, cytoplasmic fragmentation and retarded developmental rate [16, 81, 82].

Sallam et al,, concluded after conducting a series of sensitivity analyses, that assisted hatching improves the pregnancy rate, implantation rate and the ongoing pregnancy rate significantly for patients with poor prognosis treated with IVF/ICSI, particularly those with two or more previous failures [70].

This concurs with the recommendations of The Practice Committee of the American Society for Reproductive Medicine after reviewing the different published reports on the role of assisted hatching in IVF suggesting that assisted hatching may be clinically useful and that individual ART programs should evaluate their own patient populations in order to determine which subgroups may benefit from the procedure. The routine or universal performance of assisted hatching in the treatment of all IVF patients appears, at this point, to be unwarranted [7].

\section{Potential risk of $\mathrm{AH}$}

The assisted hatching procedure may be associated with specific complications independent of the IVF procedure

\begin{tabular}{|c|c|c|c|c|c|}
\hline Study & \multirow[t]{2}{*}{$\begin{array}{c}\text { Assisted hatching } \\
n / \mathbb{N}\end{array}$} & \multirow{2}{*}{$\begin{array}{c}\begin{array}{c}\text { No hatching } \\
\mathbf{n} / \mathbf{N}\end{array} \\
28 / 381\end{array}$} & $\begin{array}{c}\text { OR } \\
\text { (95\%Cl Fixed) }\end{array}$ & \multirow{2}{*}{$\begin{array}{c}\begin{array}{c}\text { Weight } \\
\%\end{array} \\
45.4\end{array}$} & \multirow{2}{*}{$\begin{array}{l}\text { OR } \\
\text { (95\%CI Fixed) } \\
1.76[1.07,2.88]\end{array}$} \\
\hline Antinori et al (12) & & & $\rightarrow-$ & & \\
\hline Chao et al (18) & $17 / 155$ & $5 / 134$ & & 8.9 & $3.18[1.14,8.86]$ \\
\hline Magli et al (13) & $67 / 506$ & $19 / 418$ & & 33.6 & $3.21[1.89,5.43]$ \\
\hline Nakayama et al (14) & $21 / 312$ & $7 / 309$ & & 12.2 & $3.11[1.30,7.43]$ \\
\hline Total $(95 \% \mathrm{Cl})$ & $151 / 1349$ & $59 / 1242$ & & 100.0 & $2.53[1.85,3.47]$ \\
\hline \multicolumn{6}{|c|}{ Test for heterogeneity chi-square $=3.28 \mathrm{df}=3 \mathrm{p}=0.35$} \\
\hline
\end{tabular}

Fig. 2 Tree diagram for implantation rates in patients with $>2$ previous IVF failures undergoing assisted hatching versus those with no assisted hatching 
Table 2 Studies of assisted hatching in patients with advanced female age

\begin{tabular}{|c|c|c|c|c|c|c|c|c|c|}
\hline \multirow[t]{2}{*}{ Type of AH } & \multicolumn{2}{|c|}{ No. of patients } & \multicolumn{3}{|c|}{ Implantation rat $(\%)$} & \multicolumn{3}{|c|}{ Pregnancy rates $(\%)$} & \multirow[t]{2}{*}{ Ref. } \\
\hline & $\mathrm{AH}$ & Control & $\mathrm{AH}$ & Control & $P$ & $\mathrm{AH}$ & Control & $P$ & \\
\hline Acid Tyrode's & 38 & 28 & 22 & 6 & $<0.001$ & 58 & 21 & $<0.005$ & {$[74]$} \\
\hline Acid Tyrode's & 211 & 174 & 3.75 & 3.55 & NS & 8.9 & 5.1 & NS & {$[75]$} \\
\hline Acid Tyrode's & 45 & 42 & 11.5 & 4 & $<0.05$ & 31 & 19 & $<0.05$ & [13] \\
\hline PZD after ICSI & 22 & 22 & 4.3 & 1.6 & NS & 9.1 & 4.5 & NS & {$[76]$} \\
\hline PZD after ICS & 30 & 29 & 5.5 & 6.9 & NS & 10 & 17.2 & NS & {$[76]$} \\
\hline Acid Tyrode's & 20 & 26 & 4.8 & 3 & NS & 15 & 11.5 & NS & [76] \\
\hline
\end{tabular}

itself, including lethal damage to the embryo and damage to individual blastomeres with reduction of embryo viability. In addition, artificial manipulation of the $\mathrm{ZP}$ has been associated with an increased risk of monozygotic twinning $[83,84]$.

A hole in the zona pellucida from any technique may deprive the embryo of its protective coat, which shields it from any detrimental factors in the female reproductive tract such as toxins, micro-organisms or immune cells. Blastomeres may become trapped in smaller holes of the zona pellucida during the hatching process, and therefore, may fail to complete hatching [29].

Alternatively, blastomeres may be lost through larger holes prior to the formation of tight junctions, possibly causing monozygotic twins, mbryonic death or even vesiculation $[46,80]$

Monozygotic twins are more common in women participating in an IVF program than in the general population. In a clinical review of 2,163 cycles of IVF, six cases of identical twin pregnancies were found [85]. Three of them had been treated with acidic Tyrode's to create larger holes $(20 \mathrm{~mm})$, and two of them had undergone subzona insemination. The increasing incidence of monozygotic twins was also stated in a review of 674 transferred embryos that underwent partial zona dissection (PZD). Monozygotic twins were found in eight $(1.2 \%)$ of them, compared with none of the 559 embryos that were not hatched [83].

The higher incidence of monozygotic twins following assisted hatching may result from two reasons. First, a small narrow opening in the zona pellucida, especially that created by PZD, may trap the hatching blastocyst in a figure 8 shape. Subdivision of the blastocyst may lead to the formation of monozygotic twins [83]. The second reason is premature hatching of the blastomeres, which may result in development of another identical embryo. Human blastomeres are joined with tight junctions that become apparent only after the 6-cell stage [86]. The loose association makes it possible to lose blastomeres when a large hole is created at an early stage. Replacing PZD with chemically assisted hatching to gain complete hatching has been advised by many researchers. However, large openings in the zona pellucida may adversely increase the risk of premature blastomere hatching. It is strongly suggested that chemically assisted hatching should be used with caution so as not to induce large openings that may be implicated in the herniation of blastomeres and induction of monozygotic twins [87].

\section{Conclusion}

This review would suggest that the routine use of assisted hatching is inappropriate in view of the lack of evidence of universal benefit and the potential risks However, there is evidence of Assisted hatching benefit in certain circumstances such as in patients with a poor prognosis, including those with two or more failed IVF cycles and poor embryo quality and older women more than 38 years of age) [11]. Higher clinical pregnancy and implantation rates have been observed after assisted hatching. However, delivery rates have not significantly improved, possibly because the small sample sizes in studies reporting delivery rates have lacked sufficient power to detect a difference. This view is consistent with the American Society for Reproductive Medicine Guidelines [7].

There are many techniques in current use to assist hatching, which may differ in both efficacy and risks. Although the equipment may be expensive, the laser assisted technique would appear to have the lowest potential risk attached to it, and it is relatively simple to perform with consistency between operators. Appropriately powered, probably multicentre research is required to confirm the safest technique to use, and to identify patients that would benefit from assisted hatching before they have had repeated unsuccessful treatment cycles. In this review there is extensive and focused revision of most researches and studies concerning with $\mathrm{AH}$ in the literature. Taking into consideration the most updated one which deals with the mechanically expanding technique with emphasis on urgent need for trials to improve the potential of $\mathrm{AH}$. Proper selection of the candidates for different $\mathrm{AH}$ 
techniques is of outmost importance. There is a strong evidence supporting that Laser $\mathrm{AH}$ is considered the best technique now as regard safety and efficacy.

\section{References}

1. Edwards RG. Clinical approaches to increasing uterine receptivity during human implantation. Hum Reprod. 1995;10 Suppl 2:60-6.

2. Huisman GJ, Fauser BC, Eijkemans MJ, Pieters MH. Implantation rates after in vitro fertilization and transfer of a maximum of two embryos that have undergone three to five days in culture. Fertil Steril. 2000;73:117-22.

3. Tucker MJ, Luecke NM, Wiker SR, Wright G. Chemical removal of the outside of the zona pellucida of day 3 human embryos has no impact on implantation rate. J Assist Reprod Genet. 1993;10:187-91.

4. Antinori S, Panci C, Selman HA, Caffa B, Dani G, Versaci C. Zona thinning with the use of laser: a new approach to assisted hatching in humans. Hum Reprod. 1996;11:590-4.

5. Valojerdi MR, Eftekhari-Yazdi P, Karimian L, Ashtiani SK. Effect of laser zona pellucida opening on clinical outcome of assisted reproduction technology in patients with advanced female age, recurrent implantation failure, or frozen-thawed embryos. Fertil Steril. 2008;90:84-91.

6. Das S, Blake D, Farquhar C, Seif MMW. Assisted hatching on assisted conception (IVF and ICSI). Cochrane Database Syst Rev. 2009;(2):CD001894. doi:10.1002/14651858.CD001894.

7. Practice Committee of Society for Assisted Reproductive Technology; Practice Committee of American Society for Reproductive Medicine, The role of assisted hatching in in vitro fertilization: a review of the literature. A Committee opinion. Fertil Steril. 2008;S196-98.

8. Margalioth EJ, Ben-Chetrit A, Gal M, Eldar-Geva T. Investigation and treatment of repeated implantation failure following IVF-ET. Hum Reprod. 2006;21:3036-43.

9. Primi MP, Senn A, Montag M, Van der Ven H, Mandelbaum J, Veiga A, et al. European multicentre prospective randomized study to assess the use of assisted hatching with a diode laser and the benefit of an immunosuppressive/antibiotic treatment in different patient populations. Hum Reprod. 2004;19:2325-33.

10. Cohen J, Malter H, Fehilly C, et al. Implantation of embryos after partial opening of oocyte zona pellucida to facilitate sperm penetration. Lancet. 1988;II:162.

11. Mansour RT, Rhodes CA, Aboulghar MA, Serour GI, Kamal A. Transfer of zona-free embryos improves outcome in poor prognosis patients: a prospective randomized controlled study. Hum Reprod. 2000;15:1061-4.

12. Antinori S, Selman HA, Caffa B, Panci C, Dani GL, Versaci C. Zona opening of human embryos using a non-contact UV laser for assisted hatching in patients with poor prognosis of pregnancy. Hum Reprod. 1996;11:2488-92.

13. Magli MC, Gianaroli L, Ferraretti AP, Fortini D, Aicardi G, Montanaro $\mathrm{N}$. Rescue of implantation potential in embryos with poor prognosis by assisted zona hatching. Hum Reprod. 1998;13:1331-5.

14. Nakayama T, Fujiwara H, Yamada S, Tastumi K, Honda T, Fujii S. Clinical application of a new assisted hatching method using a piezo-micromanipulator for morphologically lowquality embryos in poor-prognosis infertile patients. Fertil Steril. 1999;71:1014-8.

15. Cohen J, Alikani M, Trowbridge J, Rosenwaks Z. Implantation enhancement by selective assisted hatching using zona drilling of human embryos with poor prognosis. Hum Reprod. 1992;7:68591.
16. Demirel LC, Evirgen O, Al-Hasani S. The role of assisted hatching in human IVF. Middle East Fert Soc J. 2002;7:6-12.

17. Tucker MJ, Morton PC, Wright G, Ingargiola PE, Sweitzer CL, Elsner CW, et al. Enhancement of outcome from intracytoplasmic sperm injection: does coculture or assisted hatching improve implantation rates? Hum Reprod. 1996;11:2434-7.

18. Chao KH, Chen SU, Chen HF, Wu MY, Yang YS, Ho HN. Assisted hatching increases the implantation and pregnancy rate of in vitro fertilization (IVF)-embryo transfer (ET), but not that of IVF-tubal ET in patients with repeated IVF failures. Fertil Steril. 1997;67:904-8.

19. Frydman N, Madoux S, Hesters L, Duvernoy C, Feyereisen E, Le $\mathrm{Du} \mathrm{A}$, et al. A randomized double-blind controlled study on the efficacy of laser zona pellucida thinning on live birth in cases of advanced female age. Hum Reprod. 2006;21:2131-5.

20. Hurst BS, Tucker KE, Awoniyi CA, Schlaff WD. Assisted hatching does not enhance IVF success in good-prognosis patients. J Assist Reprod Genet. 1998;15(2):62-4.

21. Lanzendorf SE, Nehchiri F, Mayer JF, Oehninger S, Muasher SJ. A prospective, randomized, double-blind study for the evaluation of assisted hatching in patients with advanced maternal age. Hum Reprod. 1998;13:409-13.

22. Bleil JD, Wassarman PN. Structure and function of the zona pellucida: identification and characterisation of the proteins of the mouse oocytes zona pellucida. Dev Biol. 1980;76:185-202.

23. Van Duin M, Polman JE, DeBreet IT, et al. Recombinant human zona pellucida protein zona pellucida3 produced by Chinese hamster ovary cells induces the human sperm acrosome reaction and promotes sperm-egg fusion. Biol Reprod. 1994;5:607-17.

24. Modliniski JA. The role of the zona pellucida in the development of mouse eggs in vivo. J Embryol Exp Morphol. 1970;23:539-51.

25. Trounson AO, Moore NW. The survival and development of sheep eggs following complete or partial removal of the zona pellucida. J Reprod Fertil. 1974;41:97-108.

26. Gordon JW, Dapunt U. A new mouse model for embryos with a hatching deficiency and its use to elucidate the mechanism of blastocyst hatching. Fertil Steril. 1993;59:1296-301.

27. Schiew MC, Araujo E, Asch RH, Balmaceda JP. Enzymatic characterization of zona pellucida hardening in human eggs and embryos. J Assist Reprod Genet. 1995;12:2-7.

28. Letterie GS. Assisted hatching: relative techniques and clinical outcomes. Assist Reprod Rev. 1997;8:116-25.

29. Cohen J. Assisted hatching of human embryos. J In Vitro Fertil Embryo Transf. 1991;8:179-90.

30. Cole RJ. Cinematographic observation on the blastocyst and zona pellucida of the mouse blastocyst. J Embryol Exp Morphol. 1967;17:481-90.

31. Veeck LL, Zaninovic N. Blastocyst hatching. In: Veeck LL, Zaninovic N, editors. An atlas of human blastocysts. London: Informa Healthcare; 2003. p. 159-71.

32. Gonzales DS, Jones JM, Pinyopummintr T, Carnevale EM, Ginther OJ, Shapiro SS, et al. Trophectoderm projections: a potential means for locomotion, attachment and implantation of bovine, equine and human blastocysts. Hum Reprod. 1996;11:2739-45.

33. Hirotoshi M, Hidehiko M, Noriko F, Junko M, Azusa K, Tatsuhiro $\mathrm{T}$. Relevance of the site of assisted hatching in thawed human blastocysts: a preliminary report. Fertility and Sterility, In Press, Corrected Proof, Available online 2 March 2010.

34. Cohen J, Alikani M, Malter HE, Adler A, Talansky BE, Rosenwaks Z. Partial zona dissection or subzonal sperm insemination: microsurgical fertilization alternatives based on evaluation of sperm and embryo morphology. Fertil Steril. 1991;56:696-706.

35. Cohen J, Inge KL, Suzman M, Wright G. Video cinematography of fresh and cryopreserved embryos: a retrospective analysis of embryonic morphology and implantation. Fertil Steril. 1989;51:820 7. 
36. Wright $\mathrm{G}$, Wiker S, Elsner $\mathrm{C}$, et al. Observations on the morphology of human zygotes, pronuclei and nucleoli and implications for cryopreservation. Hum Reprod. 1990;5:109-15.

37. Chan PJ. Developmental potential of human oocytes according to zona pellucida thickness. J In Vitro Fertil Embryo Transf. 1987;4:237-41.

38. Loret De Mola JR, Garside WT, Bucci J, Tureck RW, Hyener S. Analysis of the human zona pellucida during culture: correlation with diagnosis and the preovulatory hormonal environment. J Assist Reprod Genet. 1997; 14:332-6.

39. Schiewe MC, Hazeleger NL, Sclinenti C, Balmaceda JP. Physical characterisation of blastocyst hatching mechanisms by use of a mouse anti-hatching mode. Fertil Steril. 1995;63:288-94.

40. Edwards R, Mettler LE, Walters DW. Identical twins and in vitro fertilisation. J In Vitro Fertil Embryo Transf. 1986;3:114-7.

41. Liu HC, Cohen J, Alikani M, Noyes N, Rosenwaks Z. Assisted hatching facilitates earlier implantations. Fertil Steril. 1993;60:8715.

42. Liu HC, Rosenwaks Z. Early pregnancy wastage in in-vitro fertilization patients. J In Vitro Fertil Embryo Transf. 1990;8:6572 .

43. Malter HE, Cohen J. Blastocyst formation and hatching in vitro following zona drilling of mouse and human embryos. Gamete Res. 1989;24:67-80.

44. Hershlag A, Feng HL. The effect of pre-freeze assisted hatching on post-thaw survival of mouse embryos. Fertil Steril. 2005;84:1752-4.

45. Cohen JH, Mather H, Wright G, Kort H, Massey J, Mitchell D. Partial zona dissection of human oocytes when failure of zona pellucida penetration is anticipated. Hum Reprod. 1989;4:43542.

46. Cohen J, Elsner C, Kort H, et al. Impairment of hatching process following IVF in the human and improvement of implantation by assisted hatching using micromanipulation. Hum Reprod. 1990;5:7-13.

47. Khalifa EAM, Tucker MJ, Hunt P. Cruciate thinning of the zona pellucida for more successful enhancement of blastocyst hatching in the mouse. Hum Reprod. 1992;7:532-6.

48. Cohen J, Feldberg D. Effects of the size and number of zona pellucida openings on hatching and trophoblast outgrowth in the mouse embryo. Mol Reprod Dev. 1991;30:70-8.

49. Cieslak J, Ivatchnenko V, Wolf G, Sheleg S, Verlinsky Y. Three dimensional partial zona pellucida dissection for preimplantation genetic diagnosis and assisted hatching. Fertil Steril. 1999;71:30813.

50. Nijs M, Vanderzwalman P, Bertin G, et al. Pregnancies obtained after zona softening of in vitro cultured or frozen-thawed human embryos. Hum Reprod. 1992;7:82. Abstract 141, (Abstract Book 2), Abstract of the 8th Annual Meeting of the ESHRE, The Hague, Netherlands.

51. Nijs M, Vanderzwalmen P, Segal-Bertina G, et al. A monozygotic twin pregnancy after application of zona rubbing on a frozenthawed blastocyst. Hum Reprod. 1993;8:127-9.

52. Cong F, Tao L, Ben-Yu M, Guang-Lun Z, Canquan Z. Mechanically expanding the zona pellucida of human frozen thawed embryos: a new method of assisted hatching. Fertility and Sterility, In Press, Corrected Proof, Available online 26 September 2009.

53. Tadir Y. Ten years of laser-assisted gametes and embryo manipulation. Contemp Ob/Gyn. 1998;9:2-10.

54. Palankar D, Ohad S, Lewis A, Simon A, Shenker J, Penchas S. Technique for cellular microsurgery using the $193 \mathrm{~nm}$ excimer laser. Laser Surg Med. 1991;11:580-6.

55. Rink K, Delacretaz G, Salathe RP, et al. Non-contact microdrilling of Mouse zona pellucida with an objective-delivered 1.48um Diode laser. Laser Surg Med. 1996;18:52-62.
56. Blake DA, Forsberg AS, Johansson BR, Wikland M. Laser zona pellucida thinning - alternative approach to assisted hatching. Hum Reprod. 2001;16:1959-64.

57. Mantoudis E, Podsially BT, Gorgy A, Venkat G, Craft IL. A comparison between quartz, partial and total laser assisted hatching in selected infertility patients. Hum Reprod. 2001;16:2182-6.

58. Obruca A, Strohmer H, Blaschitz A, Schonickle E, Dohr G, Feichtinger W. Ultrastructural observations in human oocytes and preimplantation embryos after zona opening using an erbium: yttrium-aluminium Garnet Er: (YAG) laser. Hum Reprod. 1997;12:2242-5.

59. Germond M, Nocera D, Senn A, Rink K, Delacnetaz G, Fakan S. Microdissection of mouse and human zona pellucida using a 1.48 Am diode laser beam: efficacy and safety of the procedure. Fertil Steril. 1995;64:604-11.

60. Kanyô K, Konc J. A follow-up study of children born after noncontact laser-assisted hatching of 96 deliveries, 134 babies. Hum Reprod. 2000;15:63. Abstract 0-156, (Abstract Book 1), Abstract of the 16th Annual Meeting of the ESHRE, Bologna, Italy.

61. Hseih YY, Huang CC, Cheng TC, Chang CC, Tsai HD, Lee MS. Laser assisted hatching of embryos is better than the chemical method for enhanceing the pregnancy rate in women with advanced age. Fertil Steril. 2002;78:179-82.

62. Makrakis E, Angeli I, Agapintou K, Pappas K, Dafereras A, Pantos K. Laser verus mechanical assisted hatching: a prospective study of clinical outcomes. Fertil Steril. 2006;86:1596-600.

63. Lanzendorf SE, Ratts VS, Moley KH, Goldstein JS, Dahan MD, Odem RR. A randomized, prospective study comparing laserassisted hatching and assisted hatching using acidified medium. Fertil Steril. 2007;87:1450-7.

64. Sagoskin AW, Levy MJ, Tucker MJ, Richter KS, Widra EA. Laser assisted hatching in good pronosis patients undergoing in vitro fertilization embryo transfer: a randomized controlled trial. Fertil Steril. 2007;87:283-7.

65. Huai L, Avner H, Gerald M, Matthew A. A retroprospective study comparing three different assisted hatching techniques. Fertil Steril. 2009;91:1323-5.

66. Hellebaut S, DeSutter P, Dozortseu D, Onghena A, Oian C, Dhont M. Does assisted hatching improve implantation rates after in vitro fertilisation or intracytoplasmic sperm injection in all patients? A prospective randomised study. J Assist Reprod Genet. 1996;13:19-22.

67. Hurst BS, Tucker KE, Awoniyi CA, Scwaff WD. Assisted hatching does not enhance IVF success in good prognosis patients. J Assist Reprod Genet. 1998;15:62-4.

68. Antinori S, Versaci C, Dani L, et al. Laser assisted hatching at the extremes of IVF spectrums. First cycle and after 6 cycles: a randomised prospective trial. Hum Reprod. 1999;14:122. Abstract 0-221 (Abstract Book I), Abstract of the 15th Annual Meeting of the ESHRE, Tours, France.

69. Baruffi R, Mauri AL, Peterson C, et al. Assisted hatching with a laser diode in patients $<37$ years old with no previous failure of implantation: a prospective randomised study. Hum Reprod. 1999;14:121. Abstract 0-218 (Abstract Book I), Abstract of the 15th Annual Meeting of the ESHRE, Tours, France.

70. Sallam HN, Sadek S, Agameya A. Assisted hatching-a metaanalysis of randomized controlled trials. J Assist Reprod Genet. 2003;20:332-42.

71. Lass A, Coucher C, Duffy S. 1000 initiated cycles of in-vitro fertilisation in women of 40 years old or more. Fertil Steril. 1998;70:1030-4.

72. Dokras A, Sargent IL, Ross C, Gardner RL, Barlow DU. The human blastocyst: morphology and human chorionic gonadotrophin secretion in vitro. Hum Reprod. 1991;6:1143-51. 
73. Meldrum DR. Female reproductive ageing: ovarian and uterine factors. Fertil Steril. 1993;59:1-5.

74. Schoolcraft WB, Schlenker T, Jones GS, Jones Jr HW. In vitro fertilisation in women aged 40 and older. The impact of assisted hatching. J Assist Reprod Genet. 1995;12:581-3.

75. Bieder D, Liushits A, Yonish M, Yemini Z, Mashiach S, Dor J. Assisted hatching by zona drilling of human embryos in women of advanced age. Hum Reprod. 1997;12:317-20.

76. Edirisinghe WR, Ahnonkitpanit V, Promviengchai S, et al. A study failing to determine significant benefits from assisted hatching: patients selected for advanced age, zona thickness of embryos, and previous failed attempts. J Assist Reprod Genet. 1999;16:294-301.

77. Carroll J, Depypene H, Mathews CD. Freeze-thaw induced changes of the zona pellucida explains decreased rates of fertilisation in frozen-thawed mouse oocytes. J Reprod Fertil. 1990;90:547-53.

78. Check JH, Hoover L, Nazari A, O'Shaughnessy A, Summers D. The effect of assisted hatching on pregnancy rates after frozen embryo transfer. Fertil Steril. 1996;65:254-7.

79. Tao J, Tamis R. Application of assisted hatching for 2-day-old, frozen-thawed embryo transfer in a poor-prognosis population. $\mathrm{J}$ Assist Reprod Genet. 1997;14:128-30.

80. Nagy ZP, Rienzi L, Iacobelli F, et al. Laser-assisted hatching and removal of degenerated blastomeres of frozen - thawed embryos improves pregnancy rates. Fertil Steril. 1999;S4 (Abstract 0-009).

81. Stein A, Rufas O, Amit S, et al. Assisted hatching by partial zona dissection of human pre-embryo in patients with recurrent implantation failure after in vitro fertilisation. Fertil Steril. 1995;63:838-41.

82. Montag M, van der Ven H. Laser-assisted hatching in assisted reproduction. Croat Med J. 1999;40:398-403.

83. Hershlag A, Paine T, Cooper GW, Scholl GM, Rawlinson K, Kvapil G. Monozygotic twinning associated with mechanical assisted hatching. Fertil Steril. 1999;71:144-6.

84. Schieve LA, Meikle SF, Peterson HB, Jeng G, Burnett NM, Wilcox LS. Does assisted hatching pose a risk for monozygotic twinning in pregnancies conceived through in vitro fertilization? Fertil Steril. 2000;74:288-94.

85. Alikani M, Noyes N, Cohen J, Rosenwaks Z. Monozygotic twinning in the human is associated with the zona pellucida architecture. Hum Reprod. 1994;9:1318-21.

86. Dale B, Gualtieri R, Talevi R, Tosti E, Santella L, Elder K. Intercellular communication in the early human embryo. Mol Reprod Dev. 1991;29:22-8.

87. Tzong S, Su-Ru C, Heng-Kien A, Yi-Yi C, Ko-Yi W, ChiiRuey T. Herniated blastomere following chemically assisted hatching may result in monozygotic twins. Fertil Sterilt. 2001;75:442-4. 